\title{
Using CF11 cellulose columns to inexpensively and effectively remove human DNA from Plasmodium falciparum-infected whole blood samples
}

Meera Venkatesan ${ }^{1,2}$, Chanaki Amaratunga ${ }^{3}$, Susana Campino ${ }^{4}$, Sarah Auburn ${ }^{4,5}$, Oliver Koch ${ }^{6}$, Pharath Lim³ ${ }^{3,7}$, Sambunny Uk ${ }^{7}$, Duong Socheat ${ }^{7}$, Dominic P Kwiatkowski ${ }^{4,6}$, Rick M Fairhurst ${ }^{3}$ and Christopher V Plowe ${ }^{1,2,8^{*}}$

\begin{abstract}
Background: Genome and transcriptome studies of Plasmodium nucleic acids obtained from parasitized whole blood are greatly improved by depletion of human DNA or enrichment of parasite DNA prior to next-generation sequencing and microarray hybridization. The most effective method currently used is a two-step procedure to deplete leukocytes: centrifugation using density gradient media followed by filtration through expensive, commercially available columns. This method is not easily implemented in field studies that collect hundreds of samples and simultaneously process samples for multiple laboratory analyses. Inexpensive syringes, hand-packed with CF11 cellulose powder, were recently shown to improve ex vivo cultivation of Plasmodium vivax obtained from parasitized whole blood. This study was undertaken to determine whether CF11 columns could be adapted to isolate Plasmodium falciparum DNA from parasitized whole blood and achieve current quantity and purity requirements for Illumina sequencing.
\end{abstract}

Methods: The CF11 procedure was compared with the current two-step standard of leukocyte depletion using parasitized red blood cells cultured in vitro and parasitized blood obtained ex vivo from Cambodian patients with malaria. Procedural variations in centrifugation and column size were tested, along with a range of blood volumes and parasite densities.

Results: CF11 filtration reliably produces 500 nanograms of DNA with less than 50\% human DNA contamination, which is comparable to that obtained by the two-step method and falls within the current quality control requirements for Illumina sequencing. In addition, a centrifuge-free version of the CF11 filtration method to isolate P. falciparum DNA at remote and minimally equipped field sites in malaria-endemic areas was validated.

Conclusions: CF11 filtration is a cost-effective, scalable, one-step approach to remove human DNA from P. falciparum-infected whole blood samples.

Keywords: CF11, Cellulose powder, Leukocyte depletion, Plasmodium falciparum, Malaria, Next-generation sequencing

\section{Background}

Recent technological advances have enabled next-generation genomic and transcriptomic analysis of Plasmodium falciparum from parasitized whole blood samples without the need for culturing. High-density genotyping of parasites obtained directly from patients with malaria

\footnotetext{
* Correspondence: cplowe@medicine.umaryland.edu

${ }^{1}$ Howard Hughes Medical Institute, University of Maryland School of Medicine, Baltimore, MD, USA

Full list of author information is available at the end of the article
}

has improved our understanding of population structure and genomic and transcriptional variation $[1,2]$. Highly parallel sequencing is currently being used to identify the genetic determinants of clinically relevant phenotypes including drug resistance, vaccine escape and disease severity. Importantly, genomic characterization of parasite populations in patients captures intra-host diversity and prevents the potential loss of sequence data from phenotype-conferring parasite isolates during their culture adaptation.

\section{Biomed Central}


The performance of highly parallel sequencing platforms, such as Illumina, is greatly enhanced in sample preparations with a high parasite-to-human nucleic acid ratio [3]. Such high ratios can be achieved by either selectively capturing parasite nucleic acids or by removing human material from the sample. Hybrid selection [4] using RNA 'baits' complementary to the P. falciparum genome can achieve over 40-fold enrichment of parasite DNA and can be performed at any time following DNA extraction [5]; however, at \$250 USD per sample, this technique may be prohibitively costly for epidemiological or population-level studies.

The alternative, leukocyte depletion, must be performed in field sites soon after blood collection and before transport and storage. Commercially available magnetic columns have been used to rapidly isolate parasitized red blood cells (RBCs) from uninfected RBCs and leukocytes [6]. However, magnetic purification depends on short-term culturing of patient blood to transform ring-stage parasites to haemozoin-rich trophozoites and schizonts, which requires equipment for in vitro parasite cultivation. Furthermore, parasites obtained directly from patients mature at different rates in culture, resulting in inconvenient time-points for purification. The current standard for leukocyte depletion of parasitized blood for $P$. falciparum nucleotide sequencing is a two-step process: centrifugation using Lymphoprep or another sucrose density gradient solution, followed by filtration using Plasmodipur filters [3]. This 'LP' method is effective but difficult to scale-up in field settings because it requires extensive handling and transfer of blood, training to perform sensitive steps, and costly commercial reagents and materials.

Filtration with hand-made columns has been used as an inexpensive and less time-consuming alternative for leukocyte depletion of Plasmodium-infected blood for decades [7,8]. Recently, non-woven fabric filters [9] and plastic syringes filled with CF11 cellulose powder (Whatman) [10] were shown to effectively remove leukocytes and platelets from Plasmodium vivax-infected blood, where their phagocytic and degranulating activities may interfere with some ex vivo studies. CF11 filtration has also been used to improve microarray-based transcriptome analysis of $P$. vivax-infected blood $[2,11]$. CF11 cellulose is thought to work by trapping leukocytes by size exclusion and/or interactions between cellulose hydroxyl groups and leukocyte surface molecules. In this study, laboratory-adapted $P$. falciparum clones were used to adapt CF11 columns to remove human DNA from P. falciparum-infected blood for genomic studies, compare CF11 filtration to the established LP method, and validate a centrifuge-free option for CF11 filtration. Both methods were also compared in a field setting using P. falciparum isolates obtained directly from patients with malaria. Furthermore, routine CF11 filtration of parasitized blood collected from patients in a second field site with minimal facilities was successfully implemented.

\section{Methods}

\section{Blood samples}

Experiments using laboratory-adapted P. falciparum clones were conducted at the University of Maryland, Baltimore. Blood obtained from healthy volunteers was mixed with purified P. falciparum parasites (3D7 or Dd2 clones) to achieve parasite densities of $10,000 / \mu \mathrm{l}$ and $5,000 / \mu \mathrm{l}$. These samples were stored at $4^{\circ} \mathrm{C}$ for up to two hours prior to CF11 or LP filtration.

Experiments using non-adapted $P$. falciparum isolates were conducted in August 2010 at Sampov Meas Referral Hospital, Pursat, Cambodia. Blood samples for these experiments were collected from patients with falciparum malaria who were $\geq 10$ years old and had a wide range of parasite densities $(\geq 10,000 / \mu \mathrm{l})$. These samples were held at $4^{\circ} \mathrm{C}$ for up to six hours prior to CF11 or LP filtration. Additional blood samples were collected in November to December 2010 at Lumphat District Health Centre, Ratanakiri, Cambodia. Blood samples were obtained from patients with falciparum malaria who were $\geq 1$ year old and had a wide range of parasite densities $\geq 10,000 / \mu \mathrm{l}$. These samples were held at $4^{\circ} \mathrm{C}$ for up to six hours prior to CF11 filtration. Following CF11 filtration, samples were stored on ice for up to six hours and transported on ice for up to 12 hours to Phnom Penh for centrifugation and RBC pellet storage.

All blood samples were collected in EDTA or CPDA-1 tubes. Heparin was not used as an anticoagulant as it thought to inhibit Taq polymerase during DNA amplification.

Blood samples from healthy volunteers were collected under a protocol approved by the Institutional Review Board of the University of Maryland School of Medicine. Blood samples from patients with falciparum malaria were collected under protocols approved by the Institutional Review Board of the National Institute for Allergy and Infectious Diseases and the Cambodian National Ethics Committee for Health Research (http://ClinicalTrials.gov identifiers: NCT00341003 and NCT01240603). Written, informed consent was obtained from all study participants or their parents or guardians.

\section{Leukocyte depletion}

Lymphoprep (Axis-shield, Oslo, Norway) + Plasmodipur (Euro-diagnostica, Arnhem, Netherlands) filtration ('LP') was performed as previously described [3]. CF11 columns were loosely filled to the $10-\mathrm{ml}$ mark, packed to the 5.5-ml mark with CF11 cellulose powder (Whatman, Kent, UK), and wetted with isotonic phosphate buffered 
saline (PBS), as described [10]. Initial tests indicated that plasma separation prior to CF11 filtration of blood did not affect human DNA depletion and that washing with PBS after CF11 filtration improved recovery of parasite DNA. CF11 filtration was therefore carried out as follows. Parasitized blood or blood/parasite mixtures were diluted with an equal volume of PBS. These samples were added to the CF11 column and allowed to flow through by gravity. To wash the sample, $5 \mathrm{ml}$ PBS was added to the column and allowed to pass through by gravity. A plunger was then inserted into the top of the column and the final few drops pushed through the column. The filtrates were centrifuged for 10 minutes at $1,000 \times \mathrm{g}$ and the supernatants removed. RBC pellets from all filtrates in all experiments were stored at $-20^{\circ} \mathrm{C}$ until DNA extraction. A packed CF11 column and filtration of blood diluted with PBS are shown in Figures 1A and $1 \mathrm{~B}$. The full CF11 filtration procedure is available on the WWARN website [12].

\section{Experimental design}

\section{CF11 versus $L P$ filtration, without modifications}

To compare the CF11 and LP methods for leukocyte depletion in a laboratory setting, three replicates of blood-parasite mixtures (3-ml volume; 10,000 parasites/ $\mu \mathrm{l})$ were prepared and filtered in parallel using each method

\section{Experimental design}

\section{CF11 versus $L P$ filtration, without modifications}

To compare the CF11 and LP methods for leukocyte depletion in a laboratory setting, three replicates of

A

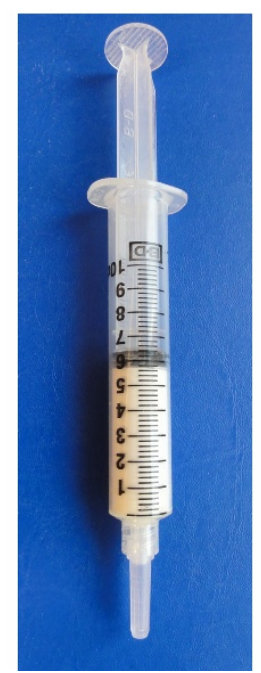

Figure 1 Packed CF11 column ready for storage, shipping or use (A) and filtration of blood-PBS mixture through CF11 column into collection tube (B). blood-parasite mixtures (3-ml volume; 10,000 parasites/ $\mu \mathrm{l})$ were prepared and filtered in parallel using each method. Unfiltered blood-parasite mixtures were prepared as control samples. To compare the two methods in a field setting (Pursat, Cambodia), $8 \mathrm{ml}$ blood from 15 patients with falciparum malaria were obtained and split into two 4-ml aliquots. Each aliquot was processed by either the CF11 or LP method.

\section{CF11 filtration, with procedural modifications}

To test a centrifuge-free method of CF11 filtration, three replicates of blood-parasite mixtures (3-ml volume; 10,000 parasites $/ \mu \mathrm{l})$ were prepared, filtered through CF11 columns, and held overnight at $4^{\circ} \mathrm{C}$ to allow RBCs pellet by gravity. Supernatants were removed the following day. To investigate the effect of decreased CF11 column length on leukocyte depletion, three replicates of blood-parasite mixtures (3-ml volume; 10,000 parasites/ $\mu \mathrm{l})$ were filtered through columns loosely filled with CF11 to the 8-ml mark and packed to the 4-ml mark.

\section{Modifications in blood volume and parasite density}

To investigate the effect of low sample volume on CF11 filtration, three replicates of 1.5 -ml blood-parasite mixtures containing 10,000 parasites/ $\mu$ l were prepared and filtered in parallel through CF11 columns loosely filled to the $5-\mathrm{ml}$ mark and packed to the $2.5-\mathrm{ml}$ mark. To investigate the effect of low parasite density on CF11 filtration, three replicates of 3-ml blood-parasite mixtures containing 5,000 parasites/ $\mu \mathrm{l}$ were prepared and filtered in parallel through CF11 columns packed to the $5.5-\mathrm{ml}$ mark. In both experiments, unfiltered blood-parasite mixtures were prepared as control samples. The effect of parasite density on CF11 and LP filtration was tested in Pursat, Cambodia, using P. falciparum isolates obtained from patients with malaria who had parasite densities ranging from 20,000-475,000/ $\mu \mathrm{l}$.

Routine blood collection for further validation and sequencing

To determine how CF11 filtration performed during a routine blood collection protocol, $3 \mathrm{ml}$ blood were obtained from 51 patients presenting with falciparum malaria in Ratanakiri, Cambodia. Blood samples were passed through CF11 columns and the filtrates were transported on ice to the laboratory in Phnom Penh, where the samples were centrifuged to obtain RBC pellets.

\section{DNA quantification}

DNA was extracted from leukocyte-depleted and unfiltered control samples using Qiamp DNA Blood Midi Kits (Qiagen, Venlo, Netherlands) according to the manufacturer's protocol. Total DNA from experiments using $P$. falciparum clones was estimated with a SpectraMax M2 Microplate Reader (Molecular Devices, Sunnyvale, 
CA, USA) using the Quant-IT PicoGreen dsDNA Assay Kit (Invitrogen, Carlsbad, CA, USA). The standard curve ranged from 0.20 to $50 \mathrm{ng} / \mu \mathrm{l}$. Total DNA from experiments using $P$. falciparum isolates was estimated with a Qubit 2.0 Fluorometer (Invitrogen, Carlsbad, CA, USA) using both the dsDNA HS and BR Assay kits.

Quantitative real-time PCR (qPCR) was used to measure the relative amounts of human and parasite DNA in each sample. Primers targeting the human LRAP (F: 5'ACGTTGGATGAATTTTCCACTGGATTCCAT-'3; R: 5'-ACGTTGGATGTGAACCATGCTCCTTGCATC-'3) and TLR9 (F: 5'-ACGTTGGATGCAAAGGGCTGG CTGTTGTAG-'3; R: 5'- ACGTTGGATGTCTACCACGAGCACTCATTC-'3) genes and the P. falciparum AMA-1 gene (F: 5'-ACGTTGGATGGATTCTCTTTCGATTTCTTTC-'3; R: 5'-ACGTTGGATGTGCTACTACTGCTTTGTCCC-'3) were used to amplify DNA from samples, negative controls and standards in duplicate. Amplification occurred in an Applied Biosystems 7300 or StepOne real-time PCR machine. Human placental and P. falciparum (3D7 clone) genomic DNA standards ranged from 0.20 to $50 \mathrm{ng} / \mu \mathrm{l}$. For each $25 \mu \mathrm{l}$ reaction, $12.5 \mu$ SYBR Green qPCR Master Mix (Applied Biosystems, Foster City, CA, USA) was mixed with $1.5 \mu \mathrm{l}$ of each forward and reverse primers at $2 \mu \mathrm{M}$ concentrations, 7.5-8.5 $\mu \mathrm{l}$ water, and 1-2 $\mu \mathrm{l}$ of template DNA. Reaction conditions were previously reported [3]: an initial denaturing step of $95^{\circ} \mathrm{C}$ for 10 minutes, five cycles of $94^{\circ}$ $\mathrm{C}$ for 45 seconds, $56^{\circ} \mathrm{C}$ for 45 seconds, $72^{\circ} \mathrm{C}$ for $45 \mathrm{sec}-$ onds, followed by 30 cycles of $94^{\circ} \mathrm{C}$ for 45 seconds, $65^{\circ} \mathrm{C}$ for 45 seconds and $72^{\circ} \mathrm{C}$ for 45 seconds and one dissociation cycle (conditions vary by real-time PCR instrument). Results were analysed using Applied Biosystems 7300 System SDS or StepOne v2.0 software.

qPCR quantification of AMA-1 and TLR9 or LRAP was used to determine the proportions of $P$. falciparum and human DNA in the sample. Total DNA was estimated by multiplying the concentration given by Spectramax or Qubit by the extracted sample volume.

\section{Statistical comparisons}

Means, variance, standard errors and statistical comparisons of means were calculated using Stata 11 software. Boxplots indicating medians, interquartile ranges and outliers were also generated using Stata 11.

\section{Results}

\section{Comparison of unmodified LP and CF11 filtration for} leukocyte depletion

Filtration of blood-parasite mixtures using unmodified LP and CF11 methods in parallel successfully depleted human DNA to $1 \%$ and $6 \%$ of total DNA, respectively (Figure 2A), well below the current Illumina sequencing threshold of $<50 \%$. CF11 was more effective than LP in

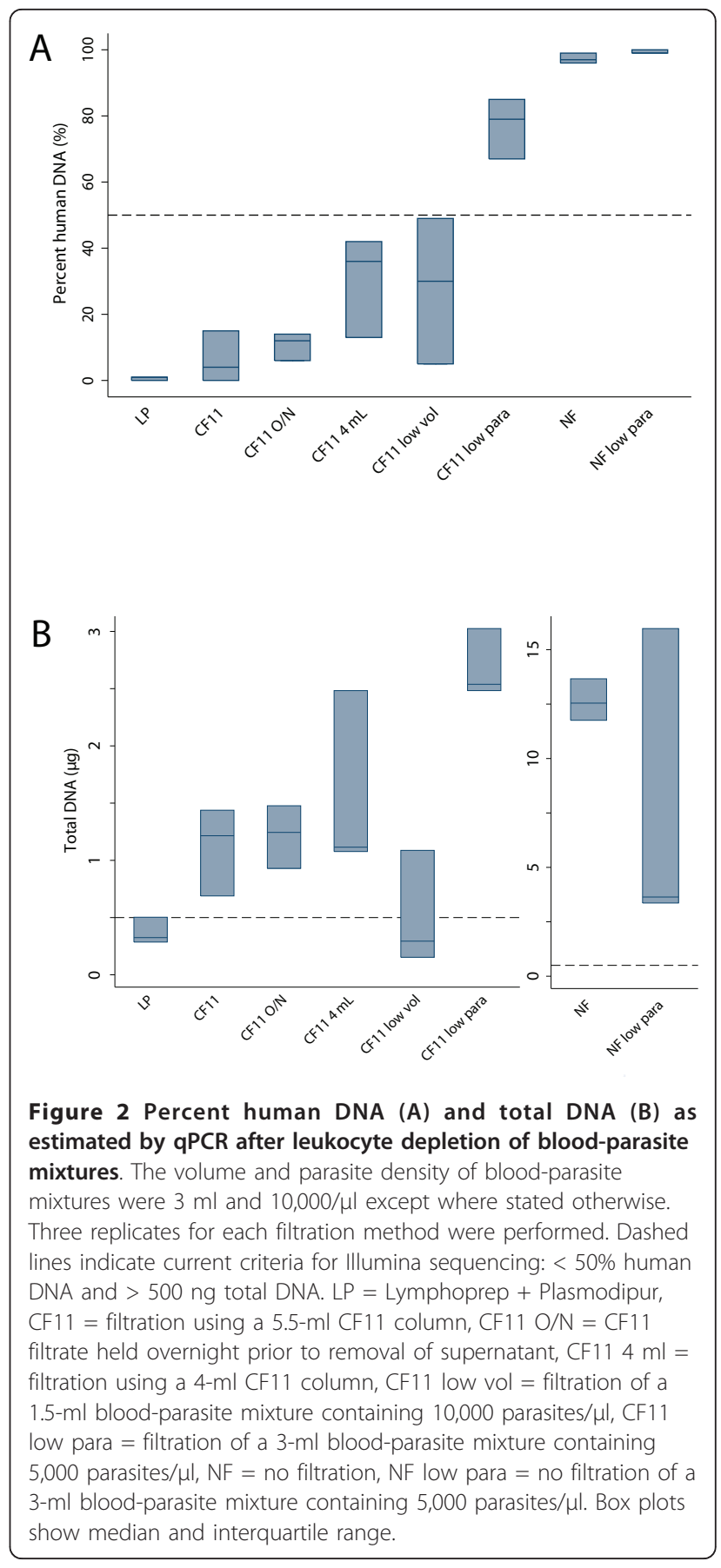

recovering total DNA from blood-parasite mixtures (mean $1.11 \mu \mathrm{g} v s .0 .37 \mu \mathrm{g} ; \mathrm{p}=0.03$ ) (Figure 2B). All 15 CF11-filtered parasitized blood samples and 12 of 14 LP-filtered parasitized blood samples yielded $<50 \%$ human DNA contamination (Figure 3A). Human DNA contamination was lower for parasitized blood filtered by CF 11 than by LP (mean $2.4 \%$ vs. $15.9 \%$; p = 0.03) (Figure 3B). Recovery of total DNA was comparably high in both sets of samples (mean $4.64 \mu \mathrm{g}$ for CF11 vs. 


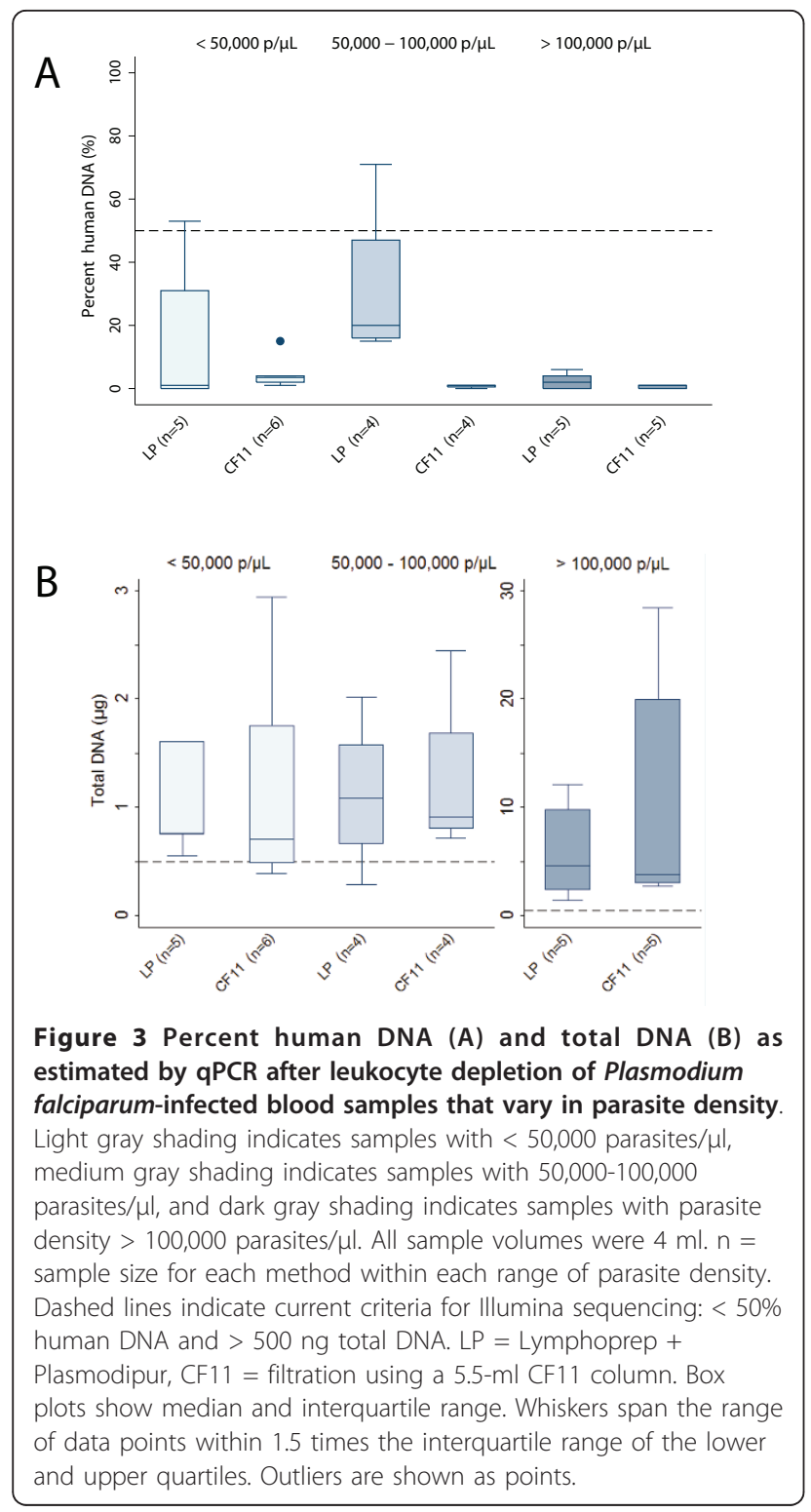

$2.86 \mu \mathrm{g}$ for LP; $\mathrm{p}=0.46)$ One LP-filtered sample failed to produce a human DNA estimate by $\mathrm{qPCR}$ and was omitted from the analysis.

\section{Procedural modifications of CF11 filtration method}

To explore whether CF11 filtrates could be processed without centrifugation, CF11-filtered blood-parasite mixtures were refrigerated overnight. The RBC pellet formed during overnight settling was not as tightly packed as that formed during centrifugation and required careful handling when removing supernatant to prevent disruption. This modification resulted in adequate human DNA depletion (mean contamination 11\%) (Figure 2A) and total DNA recovery (mean $1.22 \mu \mathrm{g}$ ) (Figure 2B). Columns with less CF11 powder, packed to the 4-ml mark rather than to the 5.5-ml mark, also yielded high total DNA (mean $1.56 \mu \mathrm{g}$ ) and a sufficiently low proportion of human DNA (mean 30\%) (Figure 2A and $2 \mathrm{~B})$.

\section{Blood volume and parasite density}

All CF11-filtered blood-parasite mixtures (3 $\mathrm{ml}$ volume, $10,000 / \mu \mathrm{l}$ parasite density) consistently yielded samples with $<30 \%$ human DNA contamination and $>500 \mathrm{ng}$ total DNA (Figure 2A and 2B). However, the ex vivo study of $P$. falciparum isolates with interesting phenotypes can be difficult if blood volumes are limited and parasite densities are low. To determine whether such limitations might significantly reduce the quality of DNA samples, CF11 filtrations were repeated using blood-parasite mixtures of lower volume $(1.5 \mathrm{ml})$ or lower parasite densities $(5,000 / \mu \mathrm{l})$. CF11 filtration of low-volume samples $(1.5 \mathrm{ml} ; 10,000$ parasites $/ \mu \mathrm{l})$ produced acceptable human DNA contamination (mean 28\%) and mean total DNA $(0.51 \mu \mathrm{g})$, but individual yields of total DNA were highly variable, ranging from 0.15 to $1.09 \mu \mathrm{g}$ (Figure 2A and $2 \mathrm{~B})$. CF11 filtration of low parasite density samples (3 ml; 5,000 parasites/ $\mu \mathrm{l}$ ) produced adequate amounts of total DNA (mean $2.68 \mu \mathrm{g}$ ) but unacceptable human DNA contamination (mean 77\%) (Figure 2A and 2B). Thus, reducing sample volumes or parasite densities by half failed to reliably produce DNA samples acceptable for Illumina sequencing.

Filtration of P. falciparum-infected blood obtained from patients with malaria (Pursat, Cambodia) using the CF11 and LP methods consistently achieved high-quality results at parasite densities ranging from 20,000 to $475,000 / \mu \mathrm{l}$ (Figure 3A and 3B). At parasite densities < $50,000 / \mu \mathrm{l}$ and $50,000-100,000 / \mu \mathrm{l}$, both filtration methods depleted human DNA contamination to $\leq 30 \%$ and produced mean DNA yields $>1 \mu$ g. Samples with $>100,000$ parasites/ $\mu$ l yielded several micrograms of DNA with < $5 \%$ human DNA contamination.

\section{Validation of routine blood collection for illumina sequencing}

The 51 parasitized blood samples that were collected and CF11-filtered in Ratanakiri, Cambodia, ranged in parasite density from 15,000 to $290,000 / \mu \mathrm{l}$ and yielded a mean $0.64 \mu \mathrm{g}$ total DNA with $34 \%$ human DNA contamination. All 51 samples met quality standards and have been successfully sequenced on the Illumina platform at the Wellcome Trust Sanger Institute (Hinxton, UK).

\section{Discussion}

As next-generation sequencing technologies improve, both costs and stringency of DNA sample quality requirements continue to fall. Between 2009 and 2011, 
requirements for Illumina sequencing of $P$. falciparum DNA by the Wellcome Trust Sanger Institute have relaxed from $30 \%$ to $50 \%$ human DNA contamination. Moreover, the possibility of sequencing multiple DNA samples in a single lane of a flow cell ('multiplex sequencing') has decreased costs immensely while yielding $\sim 2 \mathrm{~GB}$ of $P$. falciparum genomic sequence data per sample, with further improvements anticipated in coming years. To take advantage of these increasingly accessible tools, researchers need scalable procedures for DNA sample preparation in minimally equipped field sites.

This study shows that CF11 columns can be used to effectively deplete human DNA from parasitized blood and achieve Illumina sequencing requirements for total DNA yield and percent human DNA contamination, using sample volumes and parasite densities comparable to those used in LP filtration experiments [3]. A major advantage of CF11 over LP filtration is cost-effectiveness. CF11 columns cost approximately one USD each, compared to 10-50 USD for the Plasmodipur filter alone. Additionally, CF11 filtration is a very simple onestep procedure that does not require specialized equipment. A centrifugation step is only used to pellet RBCs after CF11 filtration and can be replaced with a convenient overnight period of refrigeration to allow the RBCs to pellet by gravity. These advantages should enable a large share of the malaria research community to produce parasite DNA samples appropriate for highly parallel sequencing and microarray technologies.

Because CF11 columns are hand-made rather than commercially produced, variation in the length of the column is likely to occur. Samples filtered in columns packed with less CF11 powder exhibited higher human DNA contamination but still met the threshold level for sequencing, indicating that some variation in column preparation can be tolerated. Samples with low blood volumes $(1.5 \mathrm{ml})$ or parasite densities $(5,000 / \mu \mathrm{l})$ did not consistently achieve sufficient human DNA depletion or total DNA recovery when filtered with either the CF11 or LP method. Whole-genome amplification (WGA) after DNA isolation [13] could boost the amount of starting material in cases where human DNA removal is satisfactory but total DNA yield is low. WGA may therefore be useful in enabling genomic characterization of CF11-filtered blood samples with low starting volume, such as those collected from very young children and patients with severe malarial anaemia. It may also be useful in processing blood samples with low parasite densities, as is often the case in patients with a recrudescent parasitaemia after anti-malarial treatment and those with high levels of naturally acquired immunity.

Although specific parameters have not been rigorously tested, observations in the field have indicated that
CF11 and parasitized blood can be effectively stored and used in a variety of environmental conditions. CF11 kept in an airtight container with regularly replaced desiccant and stored in a cool place can be used for at least three months, even in climates with high heat and humidity (unpublished observation). EDTA-treated blood stored at $4^{\circ} \mathrm{C}$ for up to 24 hours prior to CF11 filtration was successfully processed for Illumina DNA sequencing (unpublished observation), but for RNA analysis, no more than six hours of refrigeration is suggested (ZB Bozdech, personal communication). Updates to the range of storage conditions will be made to the WWARN protocol [12] as more information becomes available.

\section{Conclusions}

CF11 filtration currently offers the best cost-effective, one-step approach to remove human DNA from P. falciparum-infected whole blood samples and can be successfully implemented in large genome-wide sequencing studies of $P$. falciparum isolates from patients with malaria.

\section{Abbreviations}

RBC: Red Blood Cell; LP: Lymphoprep + Plasmodipur; PBS: Phosphate Buffered Saline.

\section{Acknowledgements}

The authors thank the patients from the study populations in Pursat and Ratanakiri, Cambodia, as well as the site staff members who collected and processed blood samples. The authors are grateful to Suon Seila and Sreng Sokunthea at the National Malaria Centre in Phnom Penh for their managerial and technical assistance in Pursat, Cambodia. The authors also thank Matthew Adams and Malathi Vadla at the University of Maryland, and Daniel Alcock and Eleanor Drury at the Wellcome Trust Sanger Institute, for their technical assistance in conducting laboratory experiments. This research was supported by the WorldWide Antimalarial Resistance Network, the Intramural Research Program of the National Institute of Allergy and Infectious Diseases of the US National Institutes of Health, the Howard Hughes Medical Institute, and the Wellcome Trust [098051].

\section{Author details}

${ }^{1}$ Howard Hughes Medical Institute, University of Maryland School of Medicine, Baltimore, MD, USA. ${ }^{2}$ WorldWide Antimalarial Resistance Network Molecular Module, University of Maryland School of Medicine, Baltimore, MD, USA. ${ }^{3}$ National Institute of Allergy and Infectious Diseases, National Institutes of Health, Bethesda, MD, USA. "Wellcome Trust Sanger Institute, Hinxton, Cambridge, UK. ${ }^{5}$ Global Health Division, Menzies School of Health Research, Charles Darwin University, Darwin, Australia. ${ }^{6}$ Wellcome Trust Centre for Human Genetics, University of Oxford, Oxford, UK. ${ }^{7}$ National Centre for Parasitology, Entomology and Malaria Control, Phnom Penh, Cambodia. ${ }^{8}$ Center for Vaccine Development, University of Maryland School of Medicine, Baltimore, MD, USA.

\section{Authors' contributions}

MV, CA, SC, OK, PL, SA and SU designed the study and collected the data. MV, CA and SC analysed the data. DS, DPK, RMF and CVP provided guidance and coordination for study design, data collection and analysis. MV wrote the first draft of the manuscript. RMF, CA, SC and CVP edited and revised the manuscript. All authors read and approved the final manuscript.

\section{Competing interests}

The authors declare that they have no competing interests. 


\section{References}

1. Mok S, Imwong M, Mackinnon MJ, Sim J, Ramadoss R, Yi P, Mayxay M, Chotivanich K, Liong KY, Russel B, Socheat D, Newton PN, Day NP, White NJ, Preiser PR, Nosten F, Dondorp AM, Bozdech Z: Artemisinin resistance in Plasmodium falciparum is associated with an altered temporal pattern of transcription. BMC Genomics 2011, 12:391.

2. Dharia NV, Bright AT, Westenberger SJ, Barnes SW, Batalov S, Kuhen K, Borboa R, Federe GC, McClean CM, Vinetz JM, Neyra V, Llanos-Cuentas A, Barnwell JW, Walker JR, Winzeler EA: Whole-genome sequencing and microarray analysis of ex vivo Plasmodium viva reveal selective pressure on putative drug resistance genes. Proc Natl Acad Sci USA 2010, 107:20045-20050.

3. Auburn S, Campino S, Clark TG, Djimde AA, Zongo I, Pinches R, Manske M, Mangano V, Alcock D, Anastasi E, Maslen G, Macinnis B, Rockett K, Modiano D, Newbold Cl, Doumbo OK, Ouédraogo JB, Kwiatkowski DP: An effective method to purify Plasmodium falciparu DNA directly from clinical blood samples for whole genome high-throughput sequencing. PLoS One 2011, 6:e22213.

4. Gnirke A, Melnikov A, Maguire J, Rogov P, LeProust EM, Brockman W, Fennell T, Giannoukos G, Fisher S, Russ C, Gabriel S, Jaffe DB, Lander ES, Nusbaum C: Solution hybrid selection with ultra-long oligonucleotides for massively parallel targeted sequencing. Nat Biotechnol 2009, 27:182-189.

5. Melnikov A, Galinsky K, Rogov P, Fennell T, Tyne DV, Russ C, Daniels R, Barnes KG, Bochicchio J, Ndiaye D, Sene PD, Wirth DF, Nusbaum C, Volkman SK, Birren BW, Gnirke A, Neafsey DE: Hybrid selection for sequencing pathogen genomes from clinical samples. Genome Biol 2011, 12:R73.

6. Trang DT, Huy NT, Kariu T, Tajima K, Kamei K: One-step concentration of malarial parasite-infected red blood cells and removal of contaminating white blood cells. Malar J 2004, 3:7.

7. Fulton JD, Grant PT: The sulphur requirements of the erythrocytic form of Plasmodium knowles. Biochem J 1956, 63:274-282.

8. Waki S, Suzuki M: Concentration of Plasmodium viva-infected erythrocytes. Ann Trop Med Parasitol 1986, 80:125-126.

9. Tao ZY, Xia H, Cao J, Gao Q: Development and evaluation of a prototype non-woven fabric filter for purification of malaria-infected blood. Malar J 2011, 10:251.

10. Sriprawat K, Kaewpongsri S, Suwanarusk R, Leimanis ML, Lek-Uthai U, Phyo AP, Snounou G, Russell B, Renia L, Nosten F: Effective and cheap removal of leukocytes and platelets from Plasmodium viva infected blood. Malar J 2009, 8:115.

11. Bozdech Z, Mok S, Hu G, Imwong M, Jaidee A, Russell B, Ginsburg H, Nosten F, Day NP, White NJ, Carlton JM, Preiser PR: The transcriptome of Plasmodium viva reveals divergence and diversity of transcriptional regulation in malaria parasites. Proc Natl Acad Sci USA 2008, 105:16290-16295.

12. WWARN Procedure: Leukocyte depletion of whole blood using CF11 columns v1.2. [http://www.wwarn.org/sites/default/files/WWARN\% 20Procedure_MOL02_0.pdf].

13. Wang Y, Nair S, Nosten F, Anderson TJ: Multiple displacement amplification for malaria parasite DNA. J Parasitol 2009, 95:253-255.

doi:10.1186/1475-2875-11-41

Cite this article as: Venkatesan et al: Using CF11 cellulose columns to inexpensively and effectively remove human DNA from Plasmodium falciparum-infected whole blood samples. Malaria Journal 2012 11:41

\section{Submit your next manuscript to BioMed Central and take full advantage of:}

- Convenient online submission

- Thorough peer review

- No space constraints or color figure charges

- Immediate publication on acceptance

- Inclusion in PubMed, CAS, Scopus and Google Scholar

- Research which is freely available for redistribution

Submit your manuscript at www.biomedcentral.com/submit
Biomed Central 